\title{
The cirrhotic liver is depleted of docosahexaenoic acid (DHA), a key modulator of NF-KB and TGF $\beta$ pathways in hepatic stellate cells
}

\author{
Mónica Enguita ${ }^{1,2}$, Nerea Razquin ${ }^{1,2}$, Reinald Pamplona ${ }^{3}$, Jorge Quiroga ${ }^{2,4,5}$, Jesús Prieto ${ }^{6}$ and Puri Fortes ${ }^{1,2}$
}

\begin{abstract}
Liver cirrhosis results from chronic hepatic damage and is characterized by derangement of the organ architecture with increased liver fibrogenesis and defective hepatocellular function. It frequently evolves into progressive hepatic insufficiency associated with high mortality unless liver transplantation is performed. We have hypothesized that the deficiency of critical nutrients such as essential omega-3 fatty acids might play a role in the progression of liver cirrhosis. Here we evaluated by LC-MS/MS the liver content of omega-3 docosahexaenoic fatty acid (DHA) in cirrhotic patients and investigated the effect of DHA in a murine model of liver injury and in the response of hepatic stellate cells (HSCs) (the main producers of collagen in the liver) to pro-fibrogenic stimuli. We found that cirrhotic livers exhibit a marked depletion of DHA and that this alteration correlates with the progression of the disease. Administration of DHA exerts potent anti-fibrogenic effects in an acute model of liver damage. Studies with HSCs show that DHA inhibits fibrogenesis more intensely than other omega-3 fatty acids. Data from expression arrays revealed that DHA blocks TGF $\beta$ and NF-KB pathways. Mechanistically, DHA decreases late, but not early, SMAD3 nuclear accumulation and inhibits p65/RelA-S536 phosphorylation, which is required for HSC survival. Notably, DHA increases ADRP expression, leading to the formation of typical quiescence-associated perinuclear lipid droplets. In conclusion, a marked depletion of DHA is present in the liver of patients with advanced cirrhosis. DHA displays anti-fibrogenic activities on HSCs targeting NF-KB and TGF $\beta$ pathways and inducing ADPR expression and quiescence in these cells.
\end{abstract}

\section{Introduction}

Persistent liver damage causes repeated boosts of cell death and regeneration, ultimately leading to a distortion of liver architecture known as liver cirrhosis. Cirrhosis is characterized by the formation of regenerative hepatocellular nodules surrounded by a fibrous tissue. Hepatocellular dedifferentiation and increased collagen deposition by activated hepatic stellate cells (HSCs)

\footnotetext{
Correspondence: Puri Fortes (pfortes@unav.es)

${ }^{1}$ Department of Gene Therapy and Hepatology, Center for Applied Medical

Research (CIMA), University of Navarra (UNAV), Pamplona, Spain

${ }^{2}$ Navarra Institute for Health Research (IdiSNA), Pamplona, Spain

Full list of author information is available at the end of the article.

These authors contributed equally: Jorge Quiroga, Jesús Prieto, Puri Fortes

Edited by G. Giannelli
}

constitute the pathophysiologic landmark of liver cirrhosis $^{1}$. This condition is associated with defective liver function and increased portal pressure and tends to progress to stages of more severe hepatic insufficiency and portal hypertension, resulting in complications such as portal systemic encephalopathy, gastrointestinal bleeding, malnutrition and ascite formation.

The characterization of factors that might promote cirrhosis progression is of considerable clinical interest. We have reasoned that in advanced liver cirrhosis a number of disturbances such as porto-systemic shunting, inadequate dietary intake, impaired intestinal absorption, compromised integrity of the intestinal barrier, inflammation and altered metabolism might deprive liver cells of

\section{(c) The Author(s) 2018}

(c) (i) Open Access This article is licensed under a Creative Commons Attribution 4.0 International License, which permits use, sharing, adaptation, distribution and reproduction cc) in any medium or format, as long as you give appropriate credit to the original author(s) and the source, provide a link to the Creative Commons license, and indicate if changes were made. The images or other third party material in this article are included in the article's Creative Commons license, unless indicated otherwise in a credit line to the material. If material is not included in the article's Creative Commons license and your intended use is not permitted by statutory regulation or exceeds the permitted use, you will need to obtain permission directly from the copyright holder. To view a copy of this license, visit http://creativecommons.org/licenses/by/4.0/. 
essential nutrients ${ }^{2,3}$, and that this defect could potentially influence the evolution of the disease.

Omega-3 polyunsaturated fatty acids ( $\omega 3$-PUFAs) are essential fatty acids that cannot be synthesized by the body and need to be obtained from the diet. The most relevant $\omega 3$-PUFA compounds are eicosapentaenoic acid (EPA), docosahexaenoic acid (DPA) and docosahexaenoic acid (DHA). These molecules display potent antiinflammatory effects in different conditions by inhibiting leukocyte chemotaxis and blocking the production of eicosanoids and pro-inflammatory cytokines ${ }^{4}$. Moreover, $\omega 3$-PUFAs impact cell function by influencing cell membrane composition, disrupting lipid rafts, activating G-coupled protein receptors, acting as precursors of proresolving mediators and modulating key transcription factors such as PPARs, SREBP1, ChREB, NF- $\mathrm{kB}$ and $\mathrm{LXR}^{5}$. Accordingly, hepatic DHA abundance may likely modulate the liver response to injury. However, although some studies in cirrhotic patients have shown a reduction in the circulating levels of $\omega 3$-PUFA ${ }^{6,7}$, there is no information at all regarding DHA values in the cirrhotic liver, the site where this omega- 3 would display its tissue homeostatic effects.

Fat-1 transgenic mice (which are capable of producing $\omega 3$-PUFA from $\omega 6$-PUFA) are protected against multiple liver insults such as alcohol and high-fat diet (HFD), nonalcoholic steatohepatitis (NASH) and DEN-induced $\mathrm{HCC}^{8-11}$. Moreover, oral administration of $\omega 3$-PUFA displays protective activities in several animal models of chronic hepatic damage, including NASH and $\mathrm{CCl}_{4}$-induced liver fibrosis. In these conditions $\omega 3$-PUFAs decrease steatosis, inflammation and fibrosis ${ }^{12-15}$. The anti-fibrotic effect of these compounds is in line with their ability to reduce COL1A1 expression by TGF $\beta$-stimulated $\mathrm{HSCs}^{14}$. However, the molecular mechanisms underlying this effect remain elusive.

In the present study, we show that the cirrhotic liver is strikingly depleted of DHA in parallel with the progression of the disease and that DHA reverts HSCs to quiescence and inhibits the fibrogenic responses to TGF $\beta$ stimulation and NF- $\mathrm{KB}$ activation by affecting SMAD3 nuclear accumulation and p65/RelA S536 phosphorylation.

\section{Results}

\section{DHA is deficient in cirrhotic livers}

DHA has been reported to be decreased in the plasma of cirrhotic patients ${ }^{6,7}$, but there is no information concerning its values in the cirrhotic liver, a site where DHA biological effects could be crucial in maintaining tissue homeostasis. Thus, we used liquid chromatography-tandem mass spectrometry (LC-MS/MS) to determine DHA, DPA and arachidonic acid (AA) levels in liver samples from 14 cirrhotic patients and in 12 normal liver specimens (Supplementary table 1). We found that DHA, but not AA or DPA, values were markedly reduced in the cirrhotic liver tissue (Fig. 1a and Supplementary Fig. 1). Accordingly, the ratio DHA/AA in hepatic tissue was significantly diminished in cirrhotic patients compared to controls (Fig. 1a). Interestingly, the stratification of the patients according to Child-Pugh score indicated that DHA abundance decreased in association with the progression of the disease (Fig. 1b). DHA and the ratio DHA/AA were also significantly decreased in fibrotic mouse livers compared to control livers (Fig. 1c). A similar decrease in hepatic DHA was also observed in TAA-treated fibrotic mice $\left(n=7 ;{ }^{* *} p<0.01\right.$; data not shown).

DHA has been shown to reduce oxidant stress and fibrogenesis in the liver in mouse models of NASH or chronic $\mathrm{CCl}_{4}$ administration ${ }^{14,16}$. Although DHA was found to inhibit TGF $\beta$-induced COL1A1 expression by human Lx2 stellate cells, the molecular mechanisms underpinning DHA anti-fibrogenic activity remain elusive. To better understand how DHA modulates liver fibrosis and the response to injury, we first studied the effect of DHA in a model of acute liver injury (Fig. 2a). Animals were challenged with $\mathrm{CCl}_{4}$ (days -3 and 0 ) and given by intragastric route (days $-2,-1,0$ and +1 ) DHA in sesame oil or vehicle alone, and were sacrificed at 24 and $48 \mathrm{~h}$ after the last $\mathrm{CCl}_{4}$ dose. At $24 \mathrm{~h}$ we found that hepatic DHA levels were increased in those animals given DHA but serum transaminases (ALT and AST), serum bilirubin and hepatic COL1A1, and $\alpha S M A$ mRNA levels showed similar values in both DHA-treated and -untreated mice, indicating comparable tissue damage in the two groups and no significant effect of DHA therapy at that time (Fig. 2b, c and data not shown). However, one day later, DHA-treated mice showed a marked reduction of COL1A1 and $\alpha S M A$ mRNAs and a diminished number of $\alpha$ SMA-positive cells compared to controls (Fig. 2c-e). These data suggest that DHA does not prevent hepatocellular damage but greatly attenuates the activation and fibrogenic response of HSCs in this model. Therefore, we proceeded to explore the biological effects of DHA on this cell population.

\section{DHA exceeds other w3-PUFAs in its ability to inhibit HSC activation and proliferation and to induce HSC quiescence}

In both human Lx2 stellate cells and primary mouse HSCs (mHSCs), we found that DHA reduced HSC proliferation and expression of pro-fibrogenic factors (COL1A1, $\alpha S M A$ and PDGFR $\beta$ ) in a dose-dependent manner (Supplementary Fig. 2). In addition, DHA markedly enhanced the expression of $A D R P$, a molecule that promotes lipid droplet formation (Fig. 3a). Importantly, DHA was more efficient at inducing these effects than other $\omega 3$-PUFAs (Fig. 3b, c). DHA is metabolized to lipid mediators with anti-inflammatory properties, such as Maresin, Resolvin D1 or 17(R)-Resolving D1, an aspirin-triggered epimer of Resolvin D1. However, 

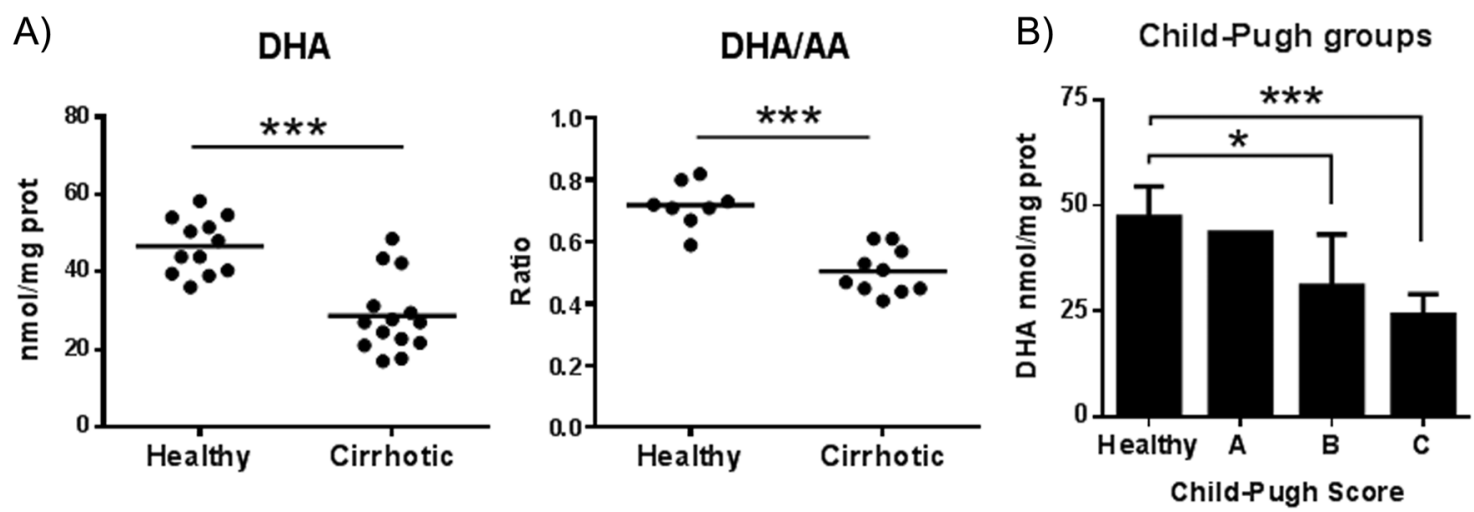

C)

DHA

DHA/AA
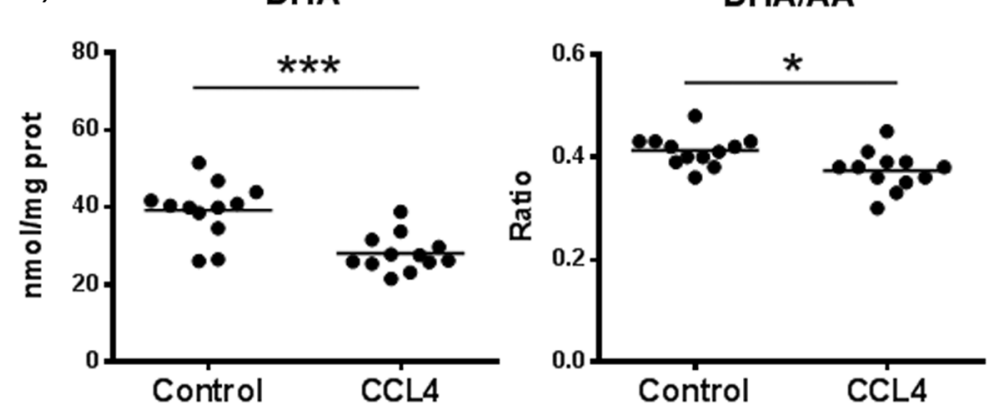

Fig. 1 DHA levels decrease in cirrhotic livers. DHA and AA levels were determined by LC-MS/MS in liver samples from healthy and cirrhotic patients (a) or in liver tissue from control or $\mathrm{CCl}_{4}$-treated mice (c). b DHA levels in patients stratified according to Child-Pugh score

treatment of Lx2 cells with these mediators did not affect the levels of COL1A1 or $\alpha S M A$ mRNAs (data not shown). As a whole, our results indicate that DHA is the strongest anti-fibrotic $\omega 3$-PUFA and that these effects could be exerted directly by DHA. Of note, Lx 2 cells treated with DHA showed a marked enrichment in cytoplasmic lipid droplets, a characteristic feature of quiescent HSCs (Fig. 3d).

Transcriptome analyses reveal that DHA modulates plural cell response pathways in both Lx2 cells and mHSCs

Ingenuity pathway analysis of microarray data obtained from mHSCs and Lx2 cells indicated that the most highly affected processes in DHA-treated cells were HSC activation/hepatic fibrosis, NRF2-mediated oxidative stress response and ATF4-mediated unfolded protein response (UPR) (Fig. 4a). The most significant upstream regulators of DHA-altered genes were TGF $\beta$ (inhibited), ATF4 (activated), TP53 and TNF $\alpha$ (Fig. 4b). Thus, our data showed that DHA downregulated pro-fibrogenic factors (COL1A1, $\alpha S M A, P D G F R \beta$ and TGF $\beta$ ) and cell survival molecules (cMET) and upregulated anti-fibrogenic and cytoprotective factors (HGF) in both mHSCs (Fig. 4c) and Lx2 cells (Supplementary Fig. 3). In addition, DHA also enhanced the expression of NRF2 target genes (such as HMOX1) and ATF4-dependent genes (such as TRIB3) (Fig. 4d). Consistently, DHA-treated Lx2 cells increased eIF2 $\alpha$ phosphorylation (an event occurring during UPR) and mobilization of NRF2 to cell nuclei (Fig. 4e, f). These observations led us to analyze whether the effects on NRF2 and UPR pathways were implicated in DHA antifibrogenic activity. With this aim, we overexpressed or knocked down (using siRNA) NFR2 in Lx2 cells and analyzed the expression of HMOX1, COL1A1 and $\alpha S M A$. We found that NFR2 overexpression enhanced HMOX1 mRNA levels but did not affect COL1A1 or $\alpha S M A$ expression. Similarly, NRF2 knockdown did not attenuate DHA-mediated inhibition of pro-fibrogenic factors (Supplementary Fig. 4a-b). Furthermore, increasing eIF2 $\alpha$ phosphorylation and thus ATF4 activation with salubrinal or inhibiting ATF4 activation with ISRIB did not affect the levels of COL1A1 or $\alpha S M A$ mRNAs and did not alter the suppressive properties of DHA on COL1A1 and $\alpha S M A$ expression (Supplementary Fig. 4c-d). These observations suggested that DHA anti-fibrogenic activity was not mediated by the NRF2 or ATF4 pathway. Therefore, we focused on the analysis of the effects of DHA on TGF $\beta$ and NF- $\mathrm{KB}$ pathways. 

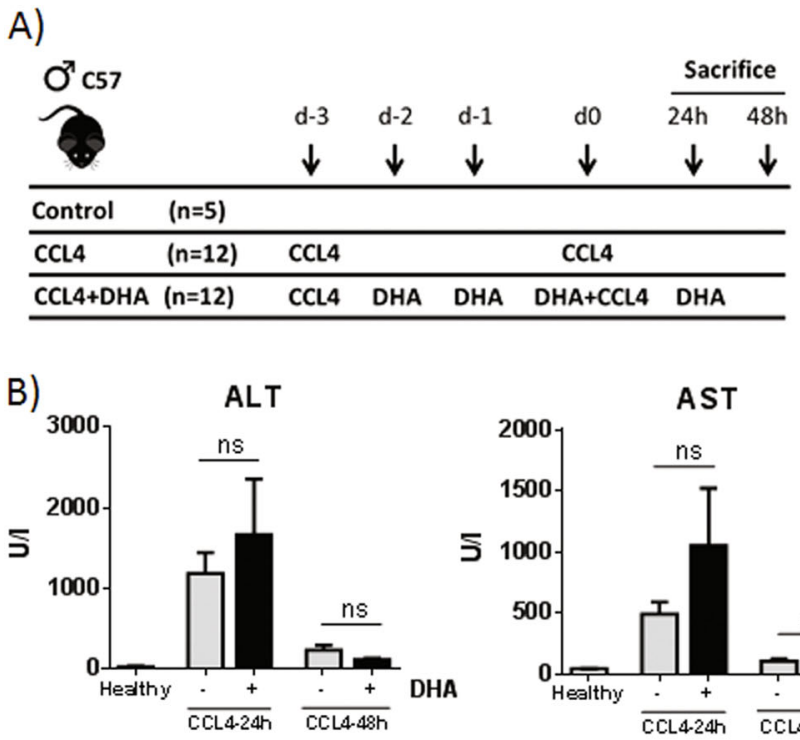

C)

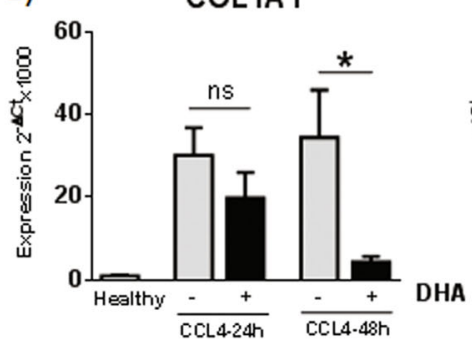

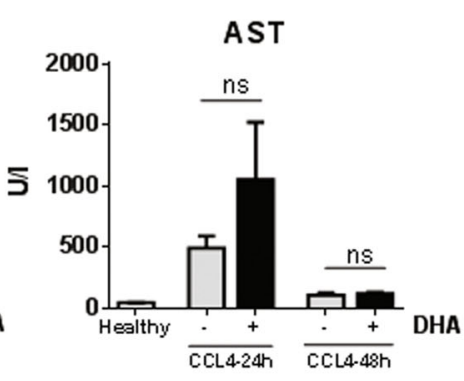

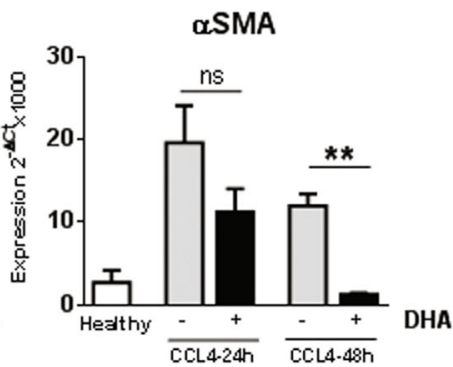

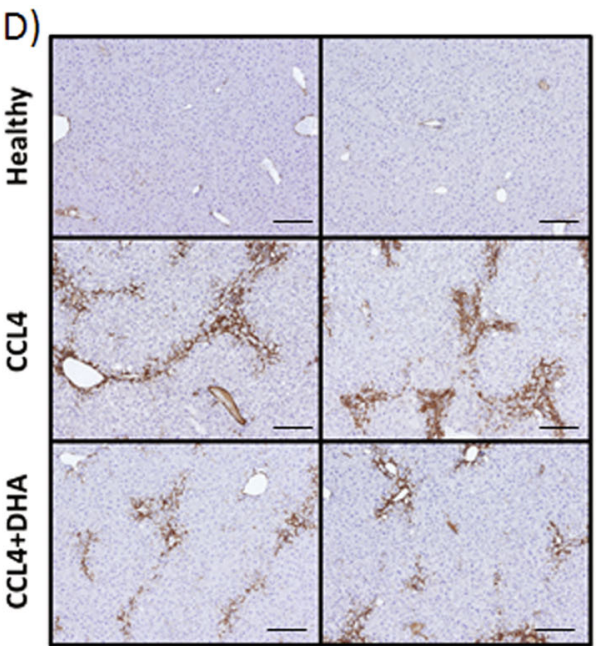

E) IHQ $\alpha$ SMA

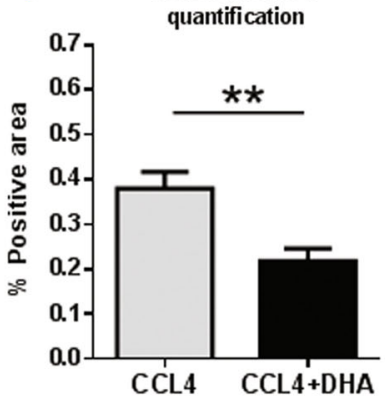

Fig. 2 DHA has anti-fibrogenic effects in an acute liver damage model. a Schematic of the experiment. b Serum ALT and AST. c COL1A1 and aSMA expression. $\mathbf{d} \mathrm{IHQ}$ of aSMA in livers from mice sacrificed $48 \mathrm{~h}$ after the last dose of $\mathrm{CCl}_{4}$. Scale bar, $100 \mu \mathrm{m}$. e Quantification of the aSMApositive area with ImageJ software. Results shown are from a representative experiment out of the three experiments performed

DHA modulates nuclear SMAD3 accumulation and abrogates the induction of TGF $\beta$ delayed target genes

As mentioned, transcriptomic analyses suggested that DHA treatment of HSCs inactivates the TGF $\beta$ pathway. The TGF $\beta$ canonical pathway requires phosphorylation of the SMAD2/3 complex, binding to SMAD4 and nuclear translocation of the hetero-oligomeric complex. We observed that DHA blocked the production of luciferase from the SBE4-Luc plasmid, which requires SMAD3 for its expression (Fig. 5a). Kinetic experiments showed that TGF $\beta$ treatment induced the phosphorylation of SMAD3 and that DHA did not affect the total levels of SMAD3 or phosphorylated SMAD3 (p-SMAD3) up to $24 \mathrm{~h}$ post TGF $\beta$ treatment (Fig. $5 b$ ). However, while the nuclear levels of SMAD3 were similar between cells incubated and those not incubated with DHA at early times $(3 \mathrm{~h})$ post TGF $\beta$ treatment, at a later time $(24 \mathrm{~h})$, a drastic decrease in the amount of nuclear SMAD3 was observed in DHAtreated cells (Fig. 5c). In agreement with these results, the expression of targets induced at early times post TGF $\beta$ treatment, such as SERPINE1/PAI-I or SMAD7, was not affected by DHA, while delayed targets, such as COL1A1 or $\alpha S M A$, showed a highly significant decrease in DHAincubated cells compared to controls (Fig. 5d). Similar results were observed at 24 and $48 \mathrm{~h}$ post TGF $\beta$ treatment (data not shown).

\section{DHA decreases phosphorylation and activity of the NF-KB subunit p65/RelA}

DHA modulates the NF- $\mathrm{kB}$ pathway in HSCs, as transcriptome analyses in DHA-treated cells showed TNF $\alpha$ as a top upstream regulator (Fig. 4). We observed that DHA drastically reduces the levels of pro-inflammatory transcripts CXCL10, CXCL9 or TNFa in Lx2 cells stimulated with TNF $\alpha$ for 6 or $40 \mathrm{~h}$ (Fig. 6a). Using total extracts from cells treated with TNF $\alpha$ for $15 \mathrm{~min}$, we observed that DHA does not affect the levels of the p65/RelA subunit of NF- $\mathrm{kB}$, but drastically decreases p65/RelA serine 536 phosphorylation (p-p65(S536)) (Fig. 6b). When nuclear and cytoplasmic fractions were evaluated, we found that most components of the NF- $\kappa B$ pathway (IKKs, ІкB $\alpha$, I $\mathrm{B} \beta$, p65, and $\mathrm{p} 50$ ) were not altered by DHA treatment 


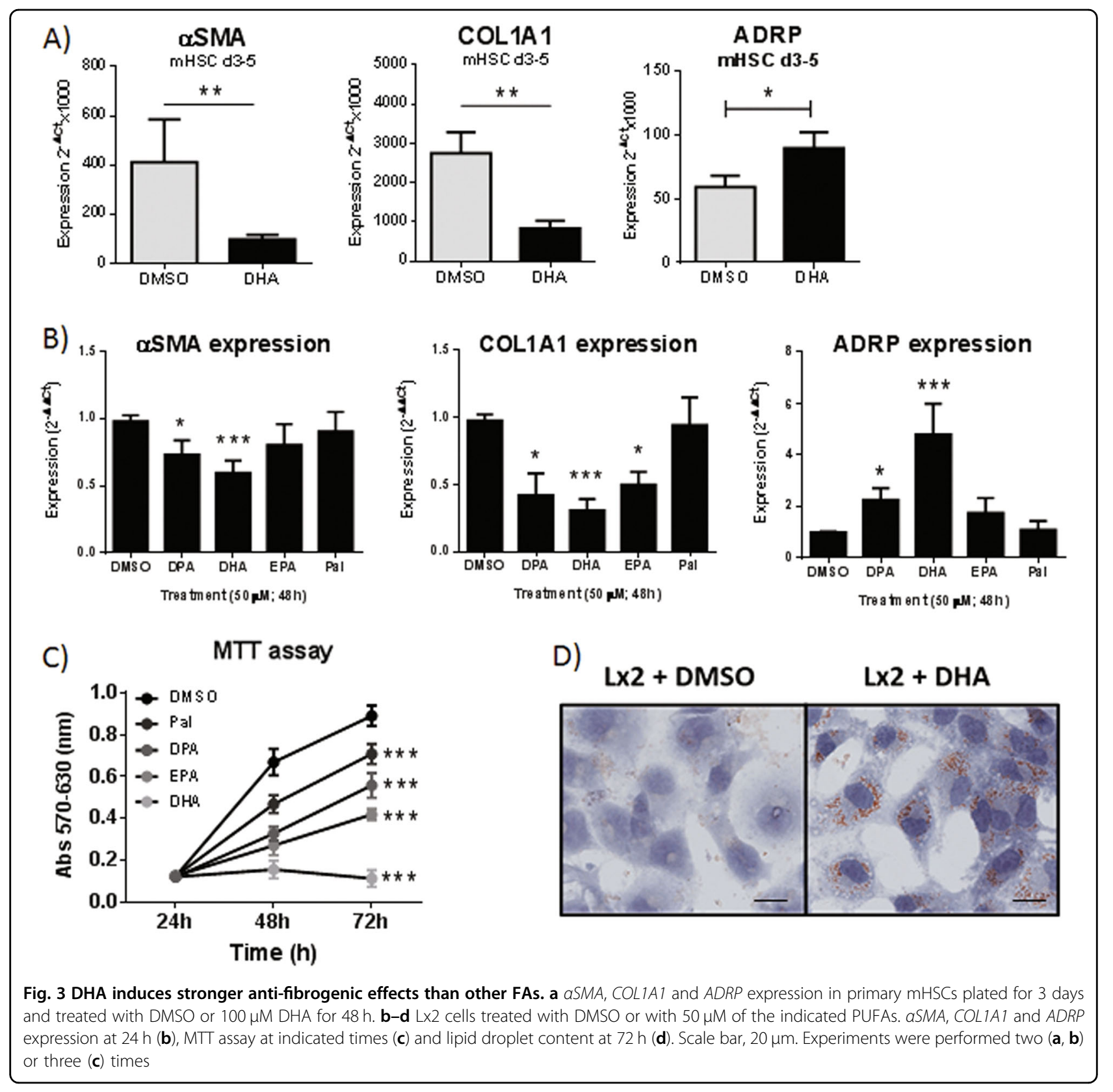

(Fig. 6c). The exception was p-p65(S536), which decreased in both nuclear and cytoplasmic extracts of DHA-treated cells. Similar results were observed in cells treated with TNF $\alpha$ for $6 \mathrm{~h}$ (data not shown).

The phosphorylation of p65(S536) can be mediated by several kinases, including IKK $\beta^{17}$. Although it has been described that DHA suppresses IKK $\beta$ phosphorylation and activity ${ }^{18}$, this is not the case in HSCs. Lx2 cells transfected with a plasmid that expresses a constitutively active IKK $\beta$ (pIKKB-ON) showed an increase in the levels of CXCL1O mRNA and other pro-inflammatory transcripts compared to control cells (Fig. $7 \mathrm{a}$ and data not shown). Surprisingly, despite the strong activation of the NF- $\mathrm{KB}$ pathway obtained under constant IKK $\beta$ activation, DHA treatment blocked the induction of these proinflammatory genes. Similar results were observed in Lx2 cells transfected with a plasmid expressing wild-type p65/RelA (Fig. 7b). Expression of a mutant S536A p65/RelA that cannot be phosphorylated does not induce CXCL10 levels but can diminish the inhibitory effect of DHA. This indicates that the regulation of p65(S536) phosphorylation by DHA is required to diminish the transcription of target genes. In agreement, western blot analyses indicated that overexpressed wild-type and 


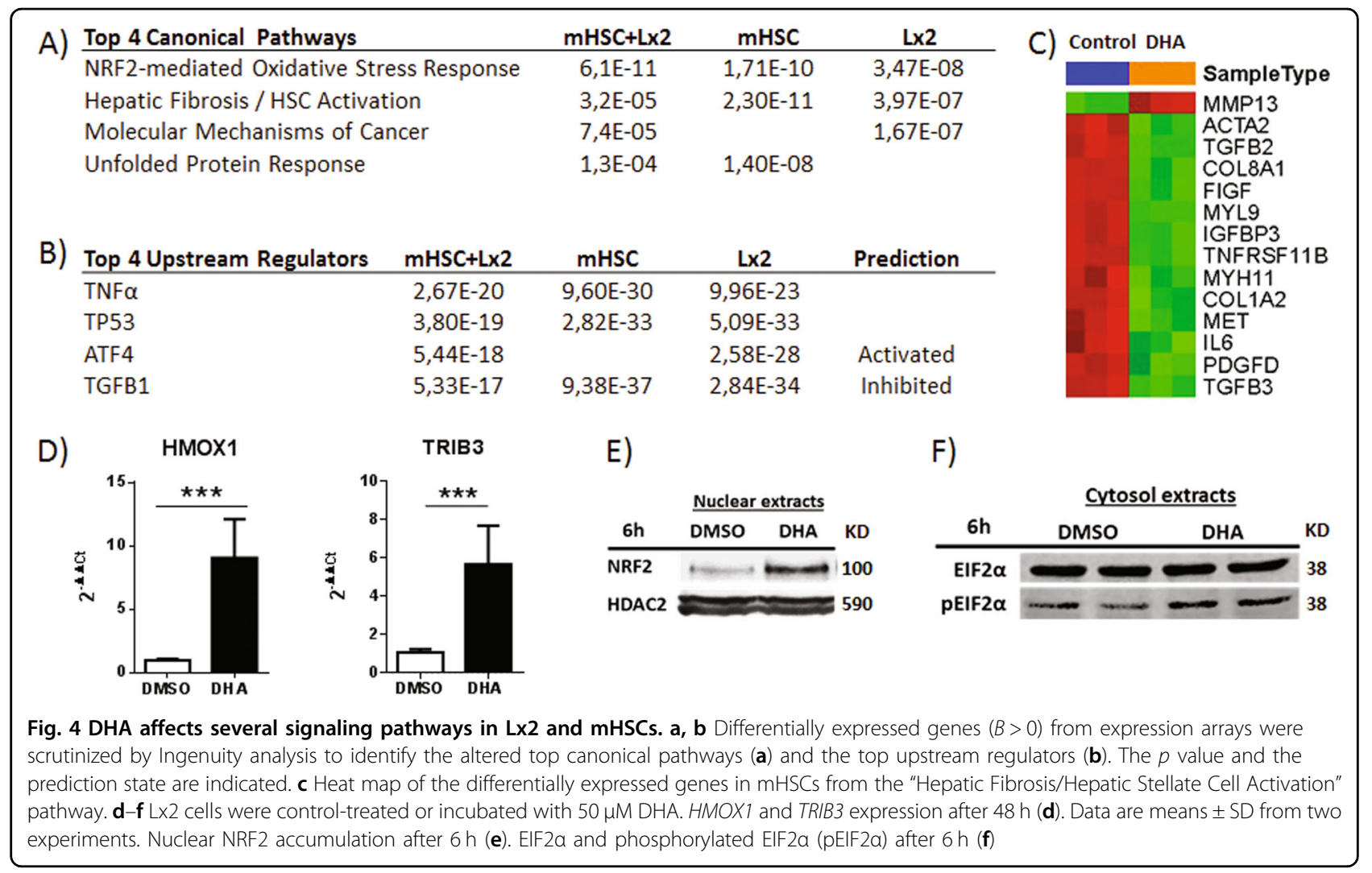

mutant p65/RelA versions are efficiently transported to the nucleus of HSCs and that DHA can dephosphorylate exogenous wild-type p65/RelA (Fig. 7c and data not shown). Interestingly, DHA-mediated regulation of p65/RelA affects the expression of pro-fibrogenic factors, as the levels of COL1A1 and $\alpha S M A$ transcripts increase in HSCs that overexpress wild-type but not S536A mutant p65/RelA (Fig. 7d). DHA treatment decreases the levels of phosphorylated p65(S536) and results in lower levels of pro-fibrogenic factors. Altogether, our results indicate that DHA inhibits the NF- $\mathrm{KB}$ pathway by decreasing $\mathrm{p} 65$ (S536) phosphorylation even under conditions of strong pathway induction by constant IKK $\beta$ activation or by the overexpression of wild-type p65/RelA. In addition, p65/ RelA activity contributes to the expression of profibrogenic factors in HSCs.

\section{Discussion}

One of the relevant findings of the present study was the presence of a profound DHA deficiency in the liver of cirrhotic patients. Notably, as liver cirrhosis progresses, liver depletion of DHA worsens in such a manner that Child-Pugh $C$ patients have about half of the values found in normal hepatic tissue. The reasons for this defect could be diverse. $\omega 3$-PUFAs cannot be synthesized by mammals and need to be obtained from the diet. Thus, although a deficient diet may be a potential cause of low liver DHA content, other pathogenic factors, including malabsorption, changes in intestinal microbiota and the presence of porto-systemic shunts, may enter into play. Indeed, in liver cirrhosis, the intestinal barrier is disturbed and the composition of gut microbiota is deeply altered the dysbiosis being more intense as the disease progresses ${ }^{19}$. It seems possible that the pro-inflammatory milieu in the gut wall and the overgrowth of pathogenic bacteria may compromise DHA bioavailability. Interestingly, it has been shown that DHA contributes to protecting the integrity of the intestinal wall and restoring gut microbiota composition ${ }^{20}$. This is a relevant observation as dysbiosis has been implicated in the pathophysiology of liver cirrhosis ${ }^{19,21}$. On the other hand, a deficiency of $\omega 3$ PUFA may enhance microbiota alteration. Another important factor contributing to liver depletion of DHA is the presence of porto-systemic shunts in patients with advanced liver cirrhosis and portal hypertension as the resulting reduction in portal blood flow to the liver may reduce hepatic inflow of crucial nutrients including DHA.

In cirrhotic patients, DHA depletion may have a significant impact on liver tissue homeostasis, likely favouring fibrosis progression. Indeed, our data in mouse models of acute $\mathrm{CCl}_{4}$-induced liver damage indicate that DHA does not reduce hepatocellular damage while it 
A)

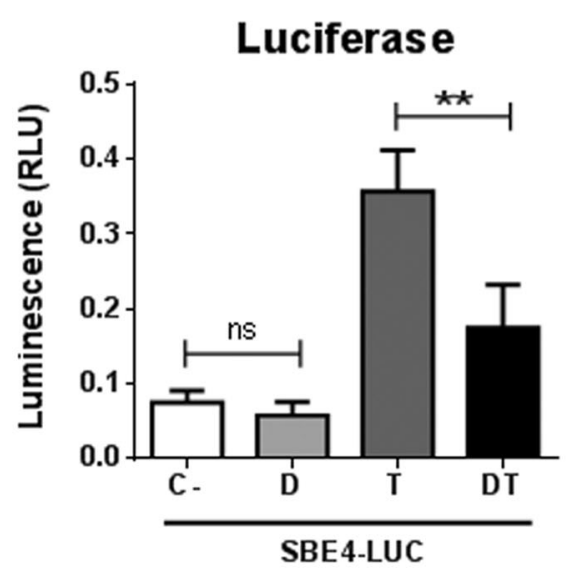

B)

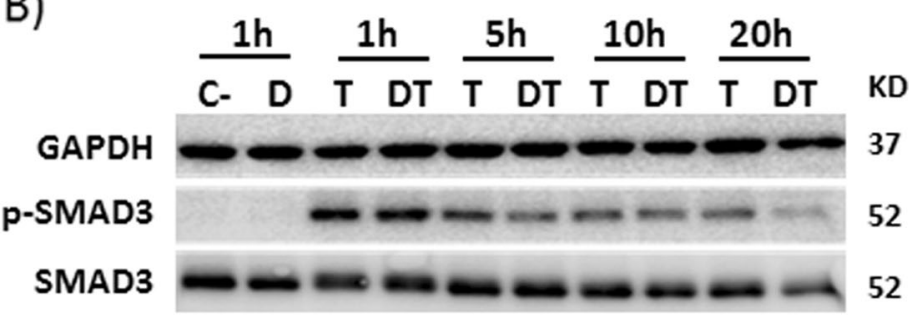

C)

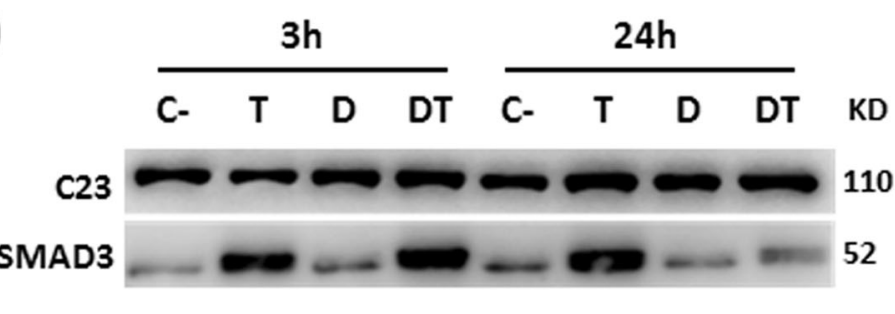

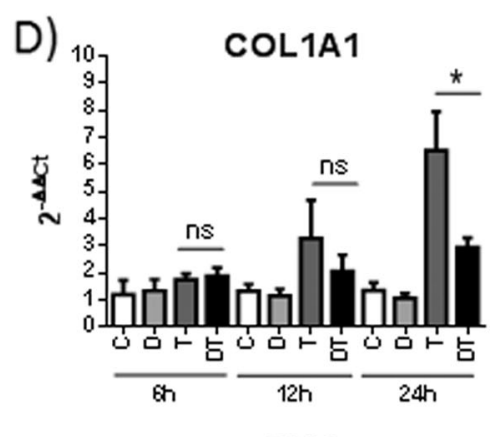
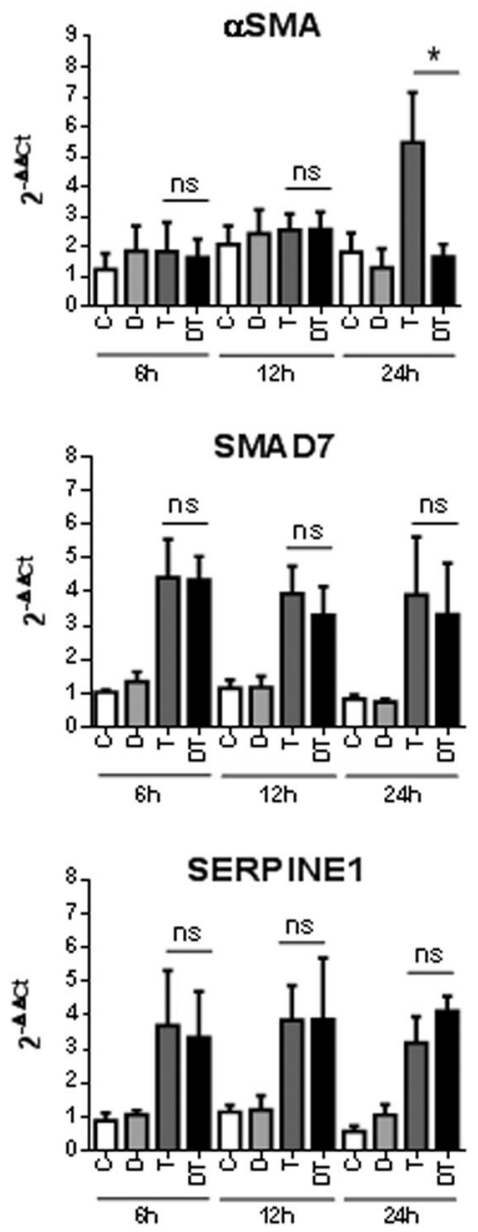

Fig. 5 DHA decreases TGF $\beta$-induced expression of COL1A1 and $a S M A$ by affecting the nuclear accumulation of SMAD3. LX2 cells treated with DMSO (C-), $30 \mu \mathrm{M}$ of DHA (D), $7.5 \mathrm{ng} / \mathrm{ml} \mathrm{TGF} \beta$ (T) or both DHA and TGF $\beta$ (DT) for the indicated times. a Luciferase levels measured $24 \mathrm{~h}$ after treatments in cells previously transfected with the SBE-Luc plasmid. Data are mean \pm SD from two experiments. $\mathbf{b}$ GAPDH, SMAD3 and p-SMAD3 proteins in total extracts. c C23 and SMAD3 proteins in nuclear extracts. d SERPINE1, SMAD7, COL1A1 and aSMA mRNA levels. Data are mean \pm SD from two experiments

strongly attenuates HSC activation and $\alpha S M A$ and COL1A1 expression. The anti-fibrogenic effects of DHA have also been shown in animal models of chronic liver damage $e^{14,16}$, and in line with these findings it has been reported that DHA dampens the response of HSCs to TGF $\beta^{14}$. However, the molecular basis underlying these effects has not been characterized.
Our transcriptional analysis of primary mHSCs and Lx2 cells showed that DHA inhibits TGF $\beta$ and NF- $\mathrm{kB}$ pathways while inducing NRF2-dependent antioxidant response and the ATF4 branch of the UPR (Fig. 4). Regarding the last two biological processes, previous studies have shown that DHA promotes nuclear accumulation of NRF2, a factor that opposes TGF $\beta$ signaling 

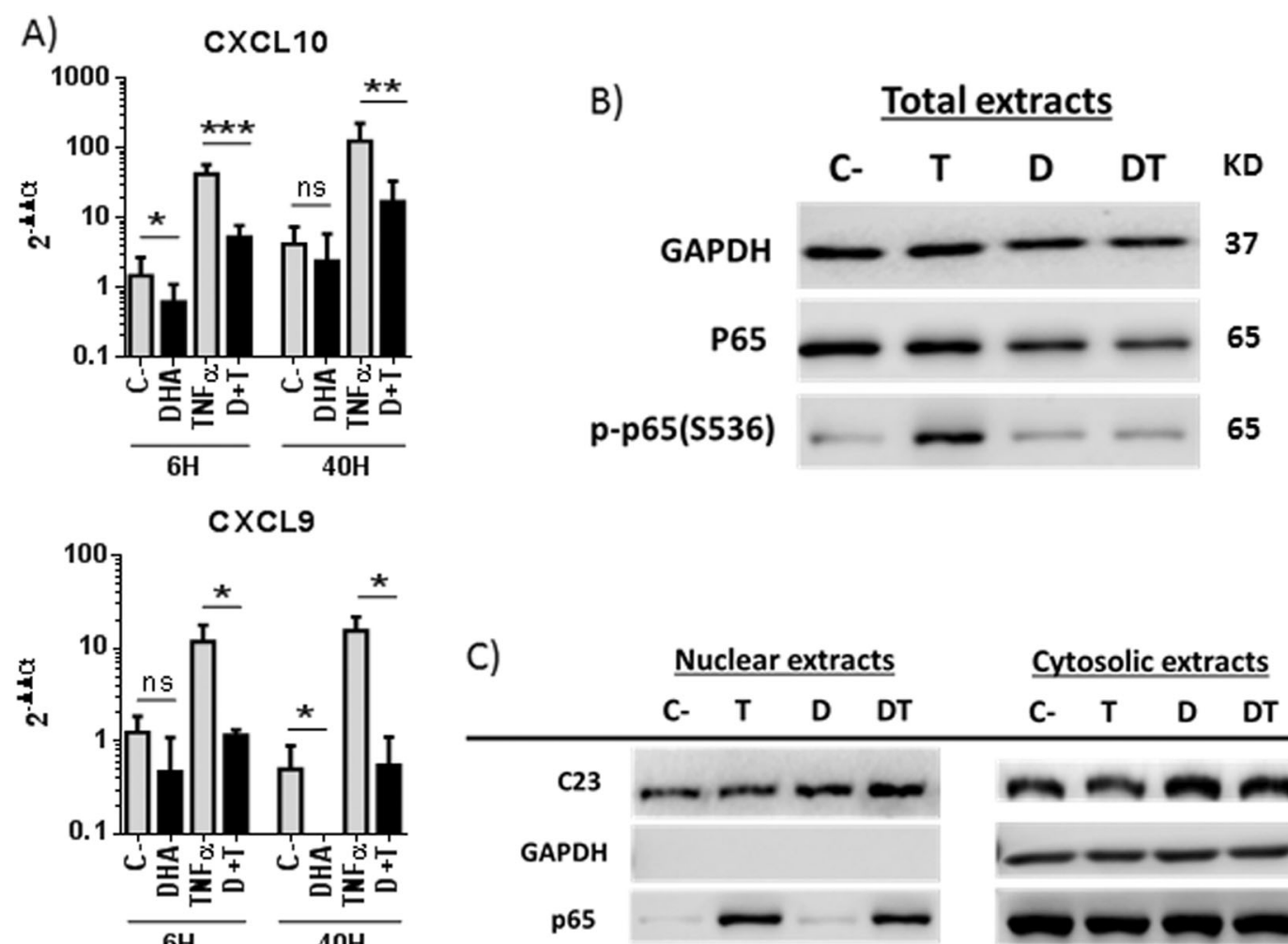

C)

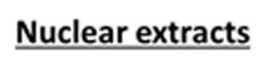

Cytosolic extracts

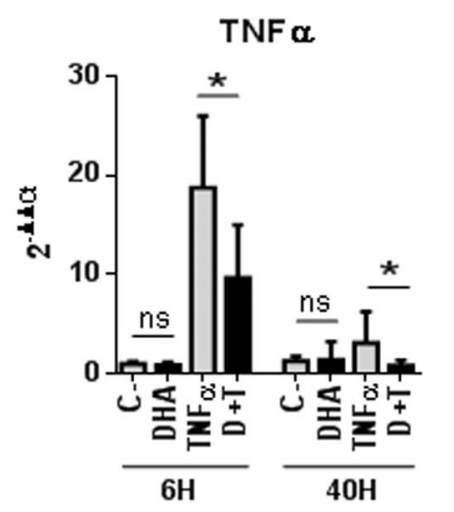

p-p65(\$536)

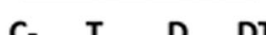

C- $\quad$ T $\quad$ D DT KD
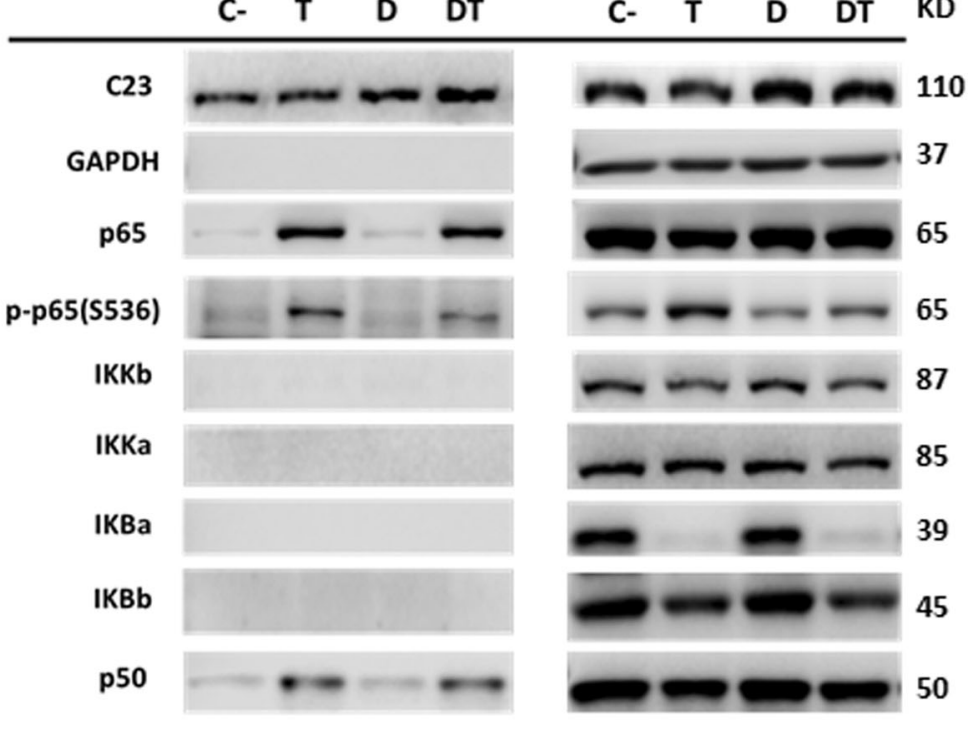

Fig. 6 DHA decreases p65/RelA Ser ${ }^{536} \mathrm{Ph}$. Lx2 cells were pretreated with DMSO or $30 \mu \mathrm{M}$ DHA for $2 \mathrm{~h}$ and then incubated or not with $35 \mathrm{ng} / \mathrm{ml}$ TNFa for $15 \mathrm{~min}(\mathbf{b}, \mathbf{c}), 6$ or $40 \mathrm{~h}$ (a). CXCL10, CXCL9 and TNFa mRNA levels (a). Data are mean \pm SD from three experiments. NF-KB signaling pathway proteins in total (b), nuclear and cytoplasmic (c) extracts. GAPDH and C23 were used as loading and fractionation controls

by binding to SMADs and inhibiting transactivation ${ }^{22-26}$. Further, DHA has also been reported to cause ER stress and eIF $2 \alpha$ phosphorylation ${ }^{27}$, and this effect may stimulate fibrogenesis ${ }^{28-30}$. However, in experiments directed to blocking or enhancing these two pathways we found that neither NRF2 nor ATF4 mediates in a significant manner the suppressive effect of DHA on COL1A1 and $\alpha S M A$ expression by HSCs. Thus, it seems plausible that DHA may deploy its anti-fibrogenic activity by interfering with TGF $\beta$ and NF- $\mathrm{kB}$ signaling.
TGF $\beta$ is a potent inducer of pro-fibrogenic genes, such as COL1A1, $\alpha S M A$ and PDGFR $\beta$, in HSCs. Moreover, TGF $\beta$ represses $A D R P$, a molecule that favors HSC quiescence and promotes lipid droplet formation by allowing the liberation of lipid drops from the ER membrane $^{31,32}$. Our transcriptome analysis indicated that DHA inhibited the TGF $\beta$ pathway, attenuated fibrogenesis and reverted HSC activation (Fig. 4a, b). In fact, one of the key genes upregulated by DHA was ADRP. Accordingly, Lx2 cells treated with DHA were found to 


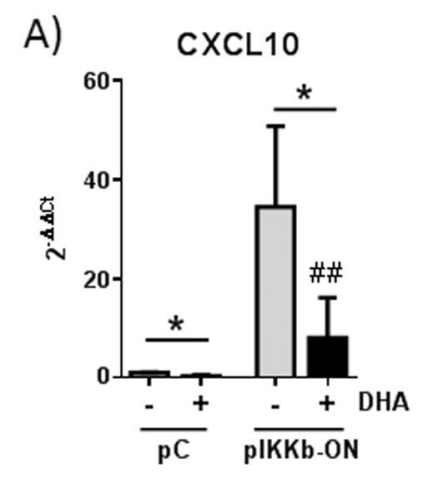

C) $\underline{\text { Total extracts }}$

B) CXCL10

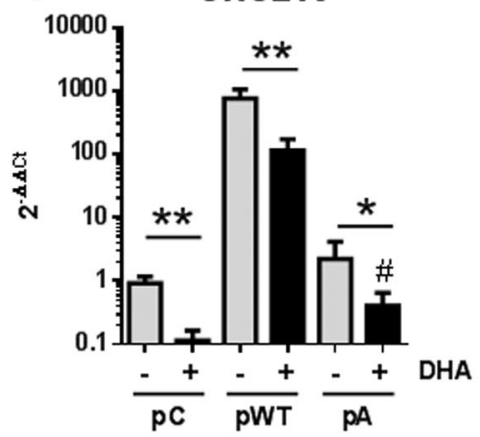

D)

p-p65(S536)

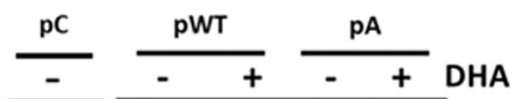

p65

GAPDH
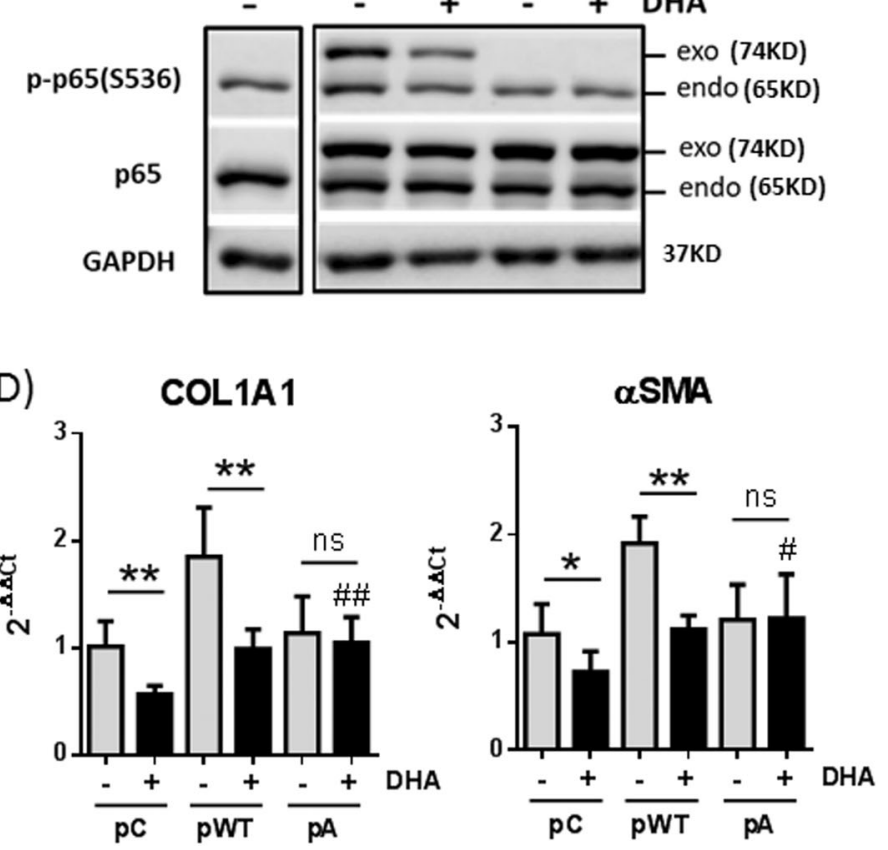

Fig. 7 Phosphorylation of p65/RelA at S536 is essential for CXCL10 induction and displays pro-fibrogenic effects in LX2 cells. a CXCL10 expression in cells transfected with a control plasmid (pC) or a plasmid expressing the constitutive active form of IKK $($ (pIKKb-ON) and mock treated or treated with $30 \mu \mathrm{M}$ DHA for $24 \mathrm{~h}$. b-d Lx2 cells were transfected with a control plasmid ( $\mathrm{pC}$ ), a plasmid expressing wild-type p65/RelA (pWT) or a mutant S536A that cannot be phosphorylated (pA). At $24 \mathrm{~h}$, the cells were mock treated or treated with $30 \mu \mathrm{M}$ DHA for $24 \mathrm{~h}$. CXCL10 (b) and COL1A1 and aSMA expression (d). Data are means \pm SD from three experiments. p65/RelA and p-p65/RelA(S536) protein in total extracts (c)

accumulate lipid drops in the cytoplasm, a characteristic feature of quiescent HSCs (Fig. 3d).

TGF $\beta$ functions by the activation and nuclear translocation of the SMAD2/3/4 factors. Then, SMADs bind the SBE (SMAD-binding element) sequence and allow the transcription of target genes. Several of these are induced very rapidly after stimulation with TGF $\beta$. These early genes have been suggested to be pure SMAD responders $^{33}$. The initial TGF $\beta$ response is followed by a secondary wave of transcription that may require cooperation of SMADs with other transcription factors such as STAT3, which help collagen expression ${ }^{34}$. Interestingly, we found that in HSCs DHA does not alter the initial transcriptional response to TGF $\beta$ but decreases the expression of the genes that are induced at $24 \mathrm{~h}$. This observation was paralleled by changes in SMAD3 content in the cell nuclei as its values were comparable to controls at $6 \mathrm{~h}$ but markedly reduced at $24 \mathrm{~h}$. This late nuclear depletion of SMAD3 by DHA causes the repression of pro-fibrogenic factors such as COL1A1 or $\alpha S M A$ (late genes) without affecting the levels of the TGF $\beta$ inhibitor SMAD7 (early gene). It should be noted that SMAD7 plays a critical role as an anti-fibrogenic factor by binding to the TGF $\beta$ receptor and blocking the association, phosphorylation and activation of the SMAD2/SMAD3 complex ${ }^{35}$.

In addition to TGF $\beta$-induced pro-fibrogenic factors, activated HSCs (aHSCs) also secrete and sense inflammatory mediators. In fact, the NF-kB pathway is required for activation and survival of HSCs and for increasing the sensitivity of these cells to TGF $\beta^{36}$. In aHSCs, TNF $\alpha$, angiotensin II, and other factors trigger IKK $\beta$, which phosphorylates p65(S536). This transcription factor leads to the expression of pro-survival molecules and fresh angiotensin II, required to maintain a positive regulatory loop that guarantees aHSC survival ${ }^{37}$. The loop results in the constitutive activation of IKK $\beta$ and constitutive levels of p-p65(S536), characteristic of aHSCs. Increased apoptosis of aHSCs is observed when this pathway is blocked by antagonists of angiotensin receptors or inhibitors of $\operatorname{IKK} \beta^{37,38}$. Here we show that DHA blocks this pathway by decreasing p65(S536) phosphorylation in an IKK $\beta$ independent manner (Figs. 6 and 7). This is different from what has been described in most cells, where DHA 
decreases the activity of IKK $\beta$ and/or reduces the phosphorylation and degradation of the negative regulator $\mathrm{I} \kappa \mathrm{B} \alpha$, thus hindering nuclear translocation of NF- $\mathrm{B}^{39-42}$. By decreasing p65(S536) phosphorylation, DHA blocks fibrosis development in different manners, including reduced production of pro-fibrogenic factors (p65 phosphorylation in S536 is required for COL1A1 and $\alpha S M A$ expression by aHSC) and induction of aHSC apoptosis (p65 phosphorylation in S536 is an essential survival factor). Finally, DHA acting on HSCs also decreases inflammation by reducing the secretion of factors such as CXCL10, a pro-inflammatory chemokine that recruits leukocytes after liver injury and contributes to aHSC survival, migration and liver fibrosis development ${ }^{43,44}$.

As mentioned, DHA has demonstrated benefits in different animal models of liver damage ${ }^{12-15}$. These data stimulated clinical trials using $\omega 3$-PUFAs (EPA or combinations of DHA and EPA) in patients with nonalcoholic fatty liver disease (NAFLD) or NASH. These studies have shown positive effects on hepatic steatosis but have not demonstrated significant and reproducible effects on fibrosis ${ }^{45-52}$. It should be noted that these clinical trials have used a mix of $\omega 3$-PUFAs at doses lower than those applied in animal studies. As DHA shows higher anti-fibrogenic activity than other $\omega 3$-PUFAs, it seems possible that using pure DHA at higher doses could be necessary to obtain anti-fibrogenic effects in patients with liver disease. Also, combinations of DHA and therapies aimed at restoring gut microbiota could afford better therapeutic results.

\section{Materials and methods}

Human samples, animal models, serum biochemistry and immunohistochemistry

Human liver samples (Supplementary Table 1) and data from patients with or without alcoholic cirrhosis were provided by the Biobank of the University of Navarra and were processed following standard operating procedures approved by the Ethical and Scientific Committees. Animal studies were performed following the regulations of the Animal Care Ethical Committee from the University of Navarra. Mice were housed 4-6 per cage in a facility in which temperature $\left(21^{\circ} \mathrm{C}\right)$, humidity and light cycle $(12 \mathrm{~h}$ light/dark) were controlled and had ad libitum access to food and water. C57 male mice (8 weeks old) were obtained from Harlan (Oxon, UK). For $\mathrm{CCl}_{4}$-induced chronic liver damage two independent experiments were performed. Liver fibrosis was developed by intraperitoneal (IP) injection of $0.75 \mu \mathrm{l} / \mathrm{g} \mathrm{CCl}_{4}$ (Merck) twice per week for $4(n=4)$ or $6(n=4)$ weeks. For TAA-induced chronic liver damage, mice received IP injections of $200 \mathrm{mg} / \mathrm{kg}$ TAA, 3 per week for 8 weeks $(n=7)$. Fibrosis was confirmed in all animals by Sirius red staining and by evaluation of COL1A1 and $\alpha S M A$ mRNA levels. Acute liver injury was induced with two doses of $1 \mu \mathrm{l} / \mathrm{g} \mathrm{CCl}_{4}(2: 5, \mathrm{v} / \mathrm{v}$ in sesame oil (Sigma S3547)) administered by IP injection (Fig. 2a). DHA treatment was performed by daily intragastric administration of $450 \mathrm{mg} / \mathrm{kg}$ DHANua (Nua Biological Innovations S.L) diluted 1:3, v/v in sesame oil and starting from $24 \mathrm{~h}$ after the first dose of $\mathrm{CCl}_{4}$. Similar results were observed when, instead of sesame oil, DHA was mixed thoroughly with phosphate buffered saline (PBS) to form an emulsion. Serum and liver samples were collected for further analyses. Serum aspartate transaminase (AST) and alanine transaminase (ALT) levels were determined (ABX diagnostics) in a Hitachi autoanalyzer (Roche). Liver tissues were fixed overnight in formaldehyde (252931.1211 Panreac), dehydrated in ethanol, and embedded in paraffin according to standard procedures. Sections were prepared and stained with a primary antibody against $\alpha$ SMA (Sigma, A2547).

\section{PUFA determination in human and mouse livers by LC-MS/MS}

Liver tissue $(25 \mathrm{mg})$ was homogenized in $100 \mu \mathrm{l}$ of PBS with $\quad 0.01 \%$ BHT (2,6-di-tert-butyl-4-methylphenol, Merck Ref. 822021). Protein concentration was determined using the Bradford assay (Bio-Rad Ref. 500-0006). Lipid extraction was carried out following the protocol described by Bligh and Dyer ${ }^{53}$. DHA and AA were quantified using multiple reaction monitoring performed in a linear ion trap triple quadrupole mass spectrometer (QTrap 4000; Sciex, Concord, Ontario, Canada) coupled with an electrospray ionization source to an Ekspert UltraHPLC 100 (Eksigent, Dublin, CA, USA) with a Spherisorb ODS2 column $(4.6 \mathrm{~mm} \times 250 \mathrm{~mm} \times 5 \mu \mathrm{m})$ (Waters). The instrument control, data acquisition, and lipid mediator quantification were performed using Analyst 1.5.2 software (Sciex). Mass spectrometry was carried out in negative ion mode using specific transitions 327/ 287 and 327/229. Calibration curves were constructed with an internal standard 332/288 and $r$ values of curves were $>0.99$ in all cases. DHA and AA (Sigma D2534 and A9673) were used as standard in the LC-MS/MS.

\section{Cell culture}

Cells were grown at $37^{\circ} \mathrm{C}$ in a $5 \% \mathrm{CO}_{2}$ atmosphere. Human Lx2 cell line was kindly provided by S. Friedman (Mount Sinai hospital, NY) and cultured in Dulbecco's Modified Eagle's Medium supplemented with $2 \mathrm{mM}$ glutamine, $100 \mu \mathrm{g} / \mathrm{ml}$ penicillin/streptomycin and $2 \%$ fetal bovine serum (FBS). Primary mHSCs were isolated from 16-week-old C57 healthy mice as described ${ }^{54}$. Briefly, mouse livers were digested with Pronase E (Sigma 000000010165921001) and Collagenase P (Sigma C5138) using a perfusion system. A Nycodenz (Sigma D2158) density gradient was used to separate other liver cells from HSCs, which have low density due to their high lipid 
content. Vitamin A autofluorescence and fat-storing characteristics were used to certify that the purity of the HSC population was higher than $95 \%$. Primary mHSCs were cultured in DMEN-F12, 10\% SFB, $100 \mu \mathrm{g} / \mathrm{ml}$ penicillin/streptomicin and $1 \%$ fungizone. The media was replaced every other day. When indicated, cells were incubated with TGF $\beta$ (RyD 240-B-002), TNF $\alpha$ (Prepotech 300-01 A) or the following fatty acids resuspended in DMSO (Sigma D4540): DHA (Sigma D2534), DPA (SC200786), EPA (Sigma E2011) and palmitic acid (Sigma P5585). After resuspension in DMSO, the proper dose of fatty acids was mixed thoroughly with cell media containing FBS and added to the cells.

\section{Cell proliferation assays}

Lx2 cells were plated in 96-well plates at 4000 cells/well, and the following day the treatment to be tested was added in a final volume of $100 \mu \mathrm{l}$. Cell viability was measured after 24, 48 and $96 \mathrm{~h}$ using the MTT assay. Briefly, $10 \mu \mathrm{l}$ of MTT stock solution $(5 \mathrm{mg} / \mathrm{ml})$ was added to each well and cells were incubated for $4 \mathrm{~h}$ at $37^{\circ} \mathrm{C}$ in an atmosphere of $5 \%$ of $\mathrm{O}_{2}$. Then, the medium was removed, $100 \mu \mathrm{l}$ of DMSO-Isopropanol (1:1, v/v) was added and the absorbance was measured at 540 and $630 \mathrm{~nm}$. The latter was considered background and was subtracted from the 540-nm measurement.

\section{Oil red staining}

Lx2 cells were plated on four-well chambers (LabTek) at 20000 cells/well and treated as described. The following day, cells were fixed with $10 \%$ paraformaldehyde (PFA) for 15 min, dehydrated with 1,2-propanediol (Sigma 398039) for $5 \mathrm{~min}$, stained with pre-warmed Oil Red O solution (Sigma O1516) for $10 \mathrm{~min}$ at $60^{\circ} \mathrm{C}$ and incubated with $85 \%$ 1,2-propanediol solution for $5 \mathrm{~min}$. Then, preparations were rinsed twice in distilled water, treated with Gill's or Mayer's hematoxylin to stain nuclei and washed twice in water for $3 \mathrm{~min}$.

\section{Plasmid transfections and luciferase reporter assays}

For overexpression experiments, p65 and p65-S536A plasmids were kindly provided by Carl Sasaki ${ }^{55}$ and pIKKb-ON plasmid was obtained from Addgene (Ref. 11105). Lx2 cells were seeded $24 \mathrm{~h}$ prior transfection at 170,000 cells/well in six-well plates. Transfection was done with $0.6 \mu \mathrm{g}$ total DNA mixed with $1.2 \mu \mathrm{l}$ Lipofectamine 3000 following the manufacturer's instructions (Invitrogen, New York, NY, USA). The media was replaced $24 \mathrm{~h}$ later and the overexpression was assessed by quantitative real-time polymerase chain reaction (qRTPCR) and western blot. For luciferase reporter assays, SBE4-Luc (Addgene, 16495) and pRL-CMV (Promega) were used. Lx2 cells were seeded $24 \mathrm{~h}$ prior transfection at 75,000 cells/well in 12-well plates. Transfection was done with $0.5 \mu$ l Lipofectamine 3000 mixed with $0.05 \mu \mathrm{g}$ CMVRenilla and $0.2 \mu \mathrm{g}$ pSBE-Luc. One day later cells were treated as described (DMSO, $30 \mu \mathrm{M}$ DHA, $7.5 \mathrm{ng} / \mathrm{ml}$ TGF $\beta$ ) for $24 \mathrm{~h}$, and Renilla and Firefly luciferase activities were measured using the Dual Luciferase System (Promega) according to the manufacturer's instructions in a Berthold Luminometer (Lumat LB 9507). The values obtained for Firefly luciferase were corrected for equal transfection efficiency with those for Renilla luciferase.

\section{RNA extraction and qRT-PCR}

Total RNA from tissue or cells was isolated using the Maxwell ${ }^{\circledast} 16$ LEV simply RNA Purification Kit (Promega) and reverse transcription was carried out with SuperScript II reverse transcriptase (Invitrogen). Messenger RNA levels were determined by qRT-PCR using the SYBR ${ }^{\circledast}$ Green Supermix Reagent (Bio-Rad). Gene expression was normalized to the RPLPO housekeeping gene and expressed as $2^{-\Delta C T}$ or $2^{-\Delta \Delta C T}$, when several experiments were grouped. All primer sequences are listed in Supplementary Table 2 .

\section{Expression arrays from Lx2 and primary mouse HSCs}

Lx2 cells were treated with DMSO, $50 \mu \mathrm{M}$ palmitic acid or $50 \mu \mathrm{M}$ DHA for $48 \mathrm{~h}$. Primary mHSCs were isolated from mice, cultured in six-well plates for 3 days and treated with DMSO or $100 \mu \mathrm{M}$ DHA for $48 \mathrm{~h}$. Total RNA was isolated from three independent samples using the Maxwell ${ }^{\circledR} 16$ LEV simply RNA Purification Kit (Promega), and RNA quantity and quality were determined using a nanodrop (Thermo Scientific, Waltham, MA, USA) and Bioanalyzer (Agilent Inc., Santa Clara, CA, USA). Excellent quality samples were hybridized to the Human gene 2.0 (Affymetrix) or the GeneChip ${ }^{\circledR}$ Mouse Gene 2.0 (Affymetrix). The methods used for sample preparation, hybridization and data analysis were based on the Affymetrix GeneChip Expression Analysis Manual (Affymetrix, Santa Clara, CA, USA). Microarray data normalization was performed using the quantile algorithm and analyzed as described ${ }^{56}$. The biological knowledge extraction was complemented using the Ingenuity Pathway Analysis (Ingenuity Systems, www. ingenuity.com). Differentially expressed genes $(\mathrm{B}>0)$ were scrutinized to identify the altered top canonical pathways and the top upstream regulators. All transcriptome data are available at the NCBI Gene Expression Omnibus (GEO) data repository (http://www.ncbi.nlm. nih.gov/geo).

\section{Protein extraction and western blot analyses}

For nuclear/cytoplasmic fractionation, cells were suspended in $200 \mu \mathrm{l}$ of Levrero buffer $(50 \mathrm{mM}$ Tris $\mathrm{HCl}(\mathrm{pH}$ 7.5), $1 \mathrm{mM}$ EDTA, $1 \%$ NP-40) containing protease inhibitor cocktail (Roche) and phosphatase inhibitors (10 nM 
$\mathrm{NaF}$ and $10 \mathrm{nM} \mathrm{NaVO} 4$ ) and incubated in ice for $10 \mathrm{~min}$. Nuclei were separated from the cytosol by centrifugation at $3000 \mathrm{rpm}$. Nuclei and total cells were lysed in radioimmunoprecipitation assay (RIPA) buffer with protease and phosphatase inhibitors. For western bBlot analyses, around $10-20 \mu \mathrm{g}$ protein was loaded per lane of polyacrylamide gel. After electrophoresis, proteins were transferred to polyvinylidene difluoride (PVDF) membranes using the Bio-Rad TransBlot (Bio-Rad, CA, USA). The membranes were blocked with $1 \%$ dried milk $+4 \%$ BSA in PBS and were incubated with the following primary antibodies: anti-GAPDH (2118), anti-SMAD3 (9523), anti-phospho-SMAD3 (9520), anti-p65 (8242), anti-phospho-p65-S536 (3033), anti-IKKB (2684), antiIKK $\alpha$ (2682) and anti-phospho-EIF2 $\alpha$ (9721) from Cell Signaling; anti-NRF2 (ab62352) from Abcam and antiEIF2 $\alpha$ (sc-11386), anti-HDAC2 (sc-7899), anti-IKB $\alpha$ (sc371), anti-IKB $\beta$ (sc-9130), anti-p50 (sc-7178) and antiC23 (sc-515312) from Santa Cruz Biotechnology. Primary antibodies were detected with the corresponding peroxidase-linked secondary antibodies, anti-rabbit IgG (7074, Cell Signaling) and anti-Mouse IgG (A0168, Sigma). Signal was developed with ECL-plus or ECL-ultra (Amersham) using the Odissey Li-Cor Hardware. Densitometry of the signal was carried out using Image Studio Lite v5.0 Software (LI-COR).

\section{Statistical analysis}

Statistical analyses were performed using Prism 5 (GraphPad Software). Differences between two groups were analyzed using Student's $t$ test or U-Mann Whitney, whereas differences between three or more groups were analyzed using the Kruskal-Wallis ANOVA test followed by Dunn's multiple comparisons test. MTT assays were analyzed by two-way ANOVA followed by Bonferroni's multiple comparisons test. Results are represented as mean $\pm \mathrm{SD}$. Statistical significance is indicated by $* * * *(p<0.001), * *(p<0.01)$ or $*(p<0.05)$. ns indicates nonsignificant differences.

\section{Acknowledgements}

This study was supported by European FEDER funding and grants from the Ministry of Economy (SAF2015-70971-R), Gobierno de Navarra 0011-1365-2016000308, Fundación Echevano, Fundación Unicaja, and Fondo de Investigación Sanitaria (PI16/02081), financed by the Instituto de Salud Carlos III. We particularly acknowledge the patients for their participation and the Biobank of the University of Navarra for its collaboration. We thank Carl Sasaki for RelA/p65 plasmids, Fernando Corrales and Leticia Odriozola for MS evaluation, Carlos Rodriguez and Sara Arcelus for help with $\mathrm{mHSC}$ isolation, and Victor Segura and Eli Guruceaga for bioinformatic analysis of the microarrays.

\footnotetext{
Author details

'Department of Gene Therapy and Hepatology, Center for Applied Medical Research (CIMA), University of Navarra (UNAV), Pamplona, Spain. ${ }^{2}$ Navarra Institute for Health Research (IdiSNA), Pamplona, Spain. ${ }^{3}$ Department of Experimental Medicine, University of Lleida (IRB), Lleida, Spain. ${ }^{4}$ Liver Unit, Clínica Universidad de Navarra, Pamplona, Spain. ${ }^{5}$ Centro de Investigación
}

Biomédica en Red de Enfermedades Hepáticas y Digestivas (Ciberehd), Pamplona, Spain. ${ }^{6} \mathrm{CIMA}$ /UNAV, Pamplona, Spain

Conflict of interest

The authors declare that they have no conflict of interest.

\section{Publisher's note}

Springer Nature remains neutral with regard to jurisdictional claims in published maps and institutional affiliations.

Supplementary Information accompanies this paper at (https://doi.org/ 10.1038/s41419-018-1243-0).

Received: 29 June 2018 Revised: 15 October 2018 Accepted: 16 October 2018

Published online: 08 January 2019

\section{References}

1. Sobrevals, L. et al. Insulin-like growth factor I gene transfer to cirrhotic liver induces fibrolysis and reduces fibrogenesis leading to cirrhosis reversion in rats. Hepatology 51, 912-921 (2010).

2. Bémeur, C. \& Butterworth, R. F. Nutrition in the management of cirrhosis and its neurological complications. J. Clin. Exp. Hepatol. 4, 141-150 (2014).

3. Suzuki, K. Endo, R. \& Kato, A. in Liver Cirrhosis - Update and Current Challenges (InTech, 2017). https://doi.org/10.5772/intechopen.68828.

4. Rodríguez-Cruz, M. \& Serna, D. S. Nutrigenomics of $\omega-3$ fatty acids: Regulators of the master transcription factors. Nutrition 41, 90-96 (2017).

5. Saini, R. K. \& Keum, Y.-S. Omega-3 and omega-6 polyunsaturated fatty acids: Dietary sources, metabolism, and significance - A review. Life. Sci. 203, 255-267 (2018).

6. Watanabe, A., Saito, S., Tsuchida, T., Higuchi, K. \& Okita, M. Low plasma levels of docosahexaenoic acid in patients with liver cirrhosis and its correction with a polyunsaturated fatty acid-enriched soft oil capsule. Nutrition 15, 284-288 (1999).

7. Ristić-Medić, D. et al. Abnormalities in the serum phospholipids fatty acid profile in patients with alcoholic liver cirrhosis - a pilot study. J. Clin. Biochem. Nutr. 53, 49-54 (2013).

8. Huang, W., Wang, B., Li, X. \& Kang, J. X. Endogenously elevated n-3 polyunsaturated fatty acids alleviate acute ethanol-induced liver steatosis. Biofactors 41, 453-462.

9. Kim, E.-H. H., Bae, J.-S. S., Hahm, K. B. \& Cha, J.-Y. Y. Endogenously synthesized $\mathrm{n}-3$ polyunsaturated fatty acids in fat-1 mice ameliorate high-fat diet-induced non-alcoholic fatty liver disease. Biochem. Pharmacol. 84, 1359-1365 (2012).

10. López-Vicario, C. et al. Molecular interplay between $\Delta 5 / \Delta 6$ desaturases and long-chain fatty acids in the pathogenesis of non-alcoholic steatohepatitis. Gut 63, 344-355 (2014)

11. Weylandt, K. H. et al. Suppressed liver tumorigenesis in fat-1 mice with elevated omega-3 fatty acids is associated with increased omega-3 derived lipid mediators and reduced TNF-a. Carcinogenesis 32, 897-903 (2011).

12. Shang, T. et al. Protective effects of various ratios of DHAVEPA supplementation on high-fat diet-induced liver damage in mice. Lipids Health Dis. 16, 65 (2017).

13. Suzuki-Kemuriyama, N. et al. Different effects of eicosapentaenoic and docosahexaenoic acids on atherogenic high-fat diet-induced non-alcoholic fatty liver disease in mice. PLOS ONE 11, e0157580 (2016).

14. Depner, C. M., Philbrick, K. A. \& Jump, D. B. Docosahexaenoic acid attenuates hepatic inflammation, oxidative stress, and fibrosis without decreasing hepatosteatosis in a Ldlr(-/-) mouse model of western diet-induced nonalcoholic steatohepatitis. J. Nutr. 143, 315-323 (2013).

15. Lytle, K. A., Wong, C. P. \& Jump, D. B. Docosahexaenoic acid blocks progression of western diet-induced nonalcoholic steatohepatitis in obese Ldllr-/- mice. PLOS ONE 12, e0173376 (2017).

16. Shaaban, A. A., Shaker, M. E., Zalata, K. R., El-kashef, H. A. \& Ibrahim, T. M. Modulation of carbon tetrachloride-induced hepatic oxidative stress, injury and fibrosis by olmesartan and omega-3. Chem. Biol. Interact. 207, 81-91 (2014). 
17. Christian, F., Smith, E. L. \& Carmody, R. J. The Regulation of NF-kB Subunits by Phosphorylation. Cells 5, 1-19 (2016).

18. Wang, T.-M. et al. Docosahexaenoic acid attenuates VCAM-1 expression and NF-kB activation in TNF-a-treated human aortic endothelial cells. J. Nutr. Biochem. 22, 187-194 (2011).

19. Acharya, C., Sahingur, S. E. \& Bajaj, J. S. Microbiota, cirrhosis, and the emerging oral-gut-liver axis. JCl insight 2, 1-11 (2017).

20. Costantini, L., Molinari, R., Farinon, B. \& Merendino, N. Impact of omega-3 fatty acids on the gut microbiota. Int. J. Mol. Sci. 18, 1-18 (2017).

21. Strnad, P., Tacke, F., Koch, A. \& Trautwein, C. Liver - guardian, modifier and target of sepsis. Nat. Rev. Gastroenterol. Hepatol. 14, 55-66 (2017).

22. Feng, R. et al. Endogenous n-3 fatty acids alleviate carbon-tetrachlorideinduced acute liver injury in fat-1 transgenic mice. Oxid. Med. Cell. Longev. 2016, 7962948 (2016).

23. González-Périz, A. et al. Docosahexaenoic acid (DHA) blunts liver injury by conversion to protective lipid mediators: protectin D1 and 17S-hydroxy-DHA. FASEB J. 20, 2537-2539 (2006).

24. Oh, C. J. et al. Dimethylfumarate attenuates renal fibrosis via NF-E2-related factor 2-mediated inhibition of transforming growth factor- $\beta / S m a d$ signaling. PLOS ONE 7, e45870 (2012)

25. Ryoo, I.-G., Ha, H. \& Kwak, M.-K. Inhibitory role of the KEAP1-NRF2 pathway in TGF 31 -stimulated renal epithelial transition to fibroblastic cells: a modulatory effect on SMAD signaling. PLOS ONE 9, e93265 (2014).

26. Rachakonda, G. et al. Increased cell migration and plasticity in Nrf2-deficient cancer cell lines. Oncogene 29, 3703-3714 (2010).

27. Jakobsen, C. H. et al. DHA induces ER stress and growth arrest in human colon cancer cells: associations with cholesterol and calcium homeostasis. J. Lipid Res. 49, 2089-2100 (2008).

28. Chusri, P. et al. HCV induces transforming growth factor $\beta 1$ through activation of endoplasmic reticulum stress and the unfolded protein response. Sci. Rep. $\mathbf{6}$ 22487 (2016).

29. Pozzer, D. et al. Endoplasmic reticulum oxidative stress triggers tgf-betadependent muscle dysfunction by accelerating ascorbic acid turnover. Sci. Rep. 7, 40993 (2017).

30. Tanjore, H., Lawson, W. E. \& Blackwell, T. S. Endoplasmic reticulum stress as a pro-fibrotic stimulus. Biochim. Biophys. Acta 1832, 940-947 (2013).

31. Stockert, J. et al. Reverse crosstalk of TGF $\beta$ and PPAR $\beta / \delta$ signaling identified by transcriptional profiling. Nucleic Acids Res. 39, 119-131 (2011).

32. Ohsaki, Y., Cheng, J., Suzuki, M., Fujita, A. \& Fujimoto, T. Lipid droplets are arrested in the ER membrane by tight binding of lipidated apolipoprotein B100. J. Cell. Sci. 121, 2415-2422 (2008).

33. Shimada, H., Staten, N. R. \& Rajagopalan, L. E. TGF- $\beta 1$ mediated activation of Rho kinase induces TGF- $\beta 2$ and endothelin-1 expression in human hepatic stellate cells. J. Hepatol. 54, 521-528 (2011).

34. Tang, L.-Y. et al. Transforming growth factor- $\beta$ (TGF- $\beta$ ) directly activates the JAK1-STAT3 axis to induce hepatic fibrosis in coordination with the SMAD pathway. J. Biol. Chem. 292, 4302-4312 (2017).

35. Dooley, S. et al. Hepatocyte-specific Smad7 expression attenuates TGF-betamediated fibrogenesis and protects against liver damage. Gastroenterology 135, 642-659 (2008)

36. Luedde, T. \& Schwabe, R. F. NF-kB in the liver-linking injury, fibrosis and hepatocellular carcinoma. Nat. Rev. Gastroenterol. Hepatol. 8, 108-118 (2011).

37. Oakley, F. et al. Angiotensin II activates I kappaB kinase phosphorylation of RelA at Ser 536 to promote myofibroblast survival and liver fibrosis. Gastroenterology 136, 2334-2344.e1 (2009).
38. Moles, A. et al. Inhibition of RelA-Ser536 phosphorylation by a competing peptide reduces mouse liver fibrosis without blocking the innate immune response. Hepatology 57, 817-828 (2013).

39. Yang, Y.-C. et al. Docosahexaenoic acid inhibition of inflammation is partially via cross-talk between Nrf2/heme oxygenase 1 and IKK/NF-KB pathways. 1. Nutr. Biochem. 24, 204-212 (2013).

40. Rahman, M. M., Bhattacharya, A. \& Fernandes, G. Docosahexaenoic acid is more potent inhibitor of osteoclast differentiation in RAW 264.7 cells than eicosapentaenoic acid. J. Cell. Physiol. 214, 201-209 (2008).

41. Paterniti, I. et al. Docosahexaenoic acid attenuates the early inflammatory response following spinal cord injury in mice: in-vivo and in-vitro studies. J. Neuroinflamm. 11, 6 (2014).

42. Oh, D. Y. et al. GPR120 is an omega-3 fatty acid receptor mediating potent anti-inflammatory and insulin-sensitizing effects. Cell 142, 687-698 (2010).

43. Hintermann, E., Bayer, M., Pfeilschifter, J. M., Luster, A. D. \& Christen, U. CXCL10 promotes liver fibrosis by prevention of NK cell mediated hepatic stellate cell inactivation. J. Autoimmun. 35, 424-435 (2010).

44. Zhang, $X$. et al. CXCL10 plays a key role as an inflammatory mediator and a non-invasive biomarker of non-alcoholic steatohepatitis. J. Hepatol. $6 \mathbf{6 1}$ 1365-1375 (2014)

45. Capanni, M. et al. Prolonged n-3 polyunsaturated fatty acid supplementation ameliorates hepatic steatosis in patients with non-alcoholic fatty liver disease: a pilot study. Aliment. Pharmacol. Ther. 23, 1143-1151 (2006).

46. Spadaro, L. et al. Effects of $n-3$ polyunsaturated fatty acids in subjects with nonalcoholic fatty liver disease. Dig. Liver. Dis. 40, 194-199 (2008).

47. Tanaka, N. et al. Highly purified eicosapentaenoic acid treatment improves nonalcoholic steatohepatitis. J. Clin. Gastroenterol. 42, 413-418 (2008).

48. Scorletti, E. et al. Design and rationale of the WELCOME trial: A randomised placebo controlled study to test the efficacy of purified long chainomega-3 fatty acid treatment in non-alcoholic fatty liver disease [corrected]. Contemp. Clin. Trials 37, 301-311 (2014).

49. Pastor-Clerigues, A., Marti-Bonmati, E., Milara, J., Almudever, P. \& Cortijo, J. Antiinflammatory and anti-fibrotic profile of fish oil emulsions used in parenteral nutrition-associated liver disease. PLOS ONE 9, e115404 (2014).

50. Argo, C. K. et al. Effects of n-3 fish oil on metabolic and histological parameters in NASH: a double-blind, randomized, placebo-controlled trial. J. Hepatol. 62 190-197 (2015).

51. Nogueira, M. A. et al. Omega-3 polyunsaturated fatty acids in treating nonalcoholic steatohepatitis: A randomized, double-blind, placebo-controlled trial. Clin. Nutr. 35, 578-586 (2016).

52. Hodson, L. et al. Docosahexaenoic acid enrichment in NAFLD is associated with improvements in hepatic metabolism and hepatic insulin sensitivity: a pilot study. Eur. J. Clin. Nutr. 71, 973-979 (2017).

53. Bligh, EG., Dyer, WJ. A rapid method of total lipid extraction and purification Can. J. Biochem. Physiol. 37, 911-917 (1959).

54. Weiskirchen, S., Tag, C. G., Sauer-Lehnen, S., Tacke, F. \& Weiskirchen, R. Isolation and culture of primary murine hepatic stellate cells. Methods Mol. Biol. 1627, 165-191 (2017).

55. Sasaki, C. Y., Barberi, T. J., Ghosh, P. \& Longo, D. L. Phosphorylation of RelA/p65 on serine 536 defines an I\{kappa\}B\{alpha\}-independent NF-\{kappa\}B pathway. J. Biol. Chem. 280, 34538-34547 (2005).

56. Carnero, E. et al. Long noncoding RNA EGOT negatively affects the antiviral response and favors HCV replication. EMBO Rep. 17, 1013-1028 (2016). 